\title{
Como se estuda o que não se diz: uma revisão sobre demanda oculta
}

\author{
How do we study what is not said: a review on hidden agenda \\ Cómo se estudia lo que no se dice: una revisión sobre demanda oculta
}

Antônio Augusto Dall'Agnol Modesto. Departamento de Medicina Preventiva, Faculdade de Medicina da Universidade de São Paulo (USP). São Paulo, SP, Brasil. filomedlit@yahoo.com.br (Autor correspondente)

Marcia Thereza Couto. Departamento de Medicina Preventiva, Faculdade de Medicina da Universidade de São Paulo (USP). São Paulo, SP, Brasil. marthet@usp.br

\section{Resumo}

Objetivos: Pessoas com demandas aparentemente bem delimitadas podem, muitas vezes, ter queixas ou preocupações ocultas ou tardiamente apresentadas, chamadas em inglês de hidden agenda, by the way syndrome ou doorknob syndrome ("síndrome da maçaneta"). Poucos trabalhos abordam o fenômeno em contexto clínico discutindo seus aspectos ou consequências sobre o cuidado do ponto de vista sociocultural. Uma pesquisa qualitativa sobre saúde dos homens realizada pelos autores exigiu estudar a produção científica relacionada à hidden agenda de forma descritiva e interpretativa. Métodos: Esta revisão, do tipo estado da questão, abarcou artigos que tratassem da hidden agenda (ou expressões similares) no contexto clínico geral ou especializado, médico ou multiprofissional, em português, inglês ou espanhol, no período de 2000 a 2014. Partindo de 210 resultados iniciais em 3 bases de dados internacionais, foram selecionadas 39 publicações, que foram avaliadas quanto a características objetivas e subjetivas. Resultados: Identificando os temas predominantes nas pesquisas, notamos que hidden agenda é a expressão mais específica, sendo demanda oculta sua melhor tradução. Quase todos os estudos relacionavam a atenção a demandas ocultas a melhores desfechos ou desdobramentos do atendimento, embora nenhum avaliasse essas consequências de forma aprofundada. Conclusão: A revisão deixa claro que a demanda oculta é um elemento problematizador para pensar a prática médica e potencializador para a clínica, especialmente ao buscar-se um cuidado integral e um diálogo efetivo entre profissional e usuário(a). São necessários estudos que articulem o fenômeno a aspectos socioculturais ou tecnoassistenciais e que contemplem alguma discussão sobre construção da demanda, ausente nos artigos revisados.

\section{Abstract}

Objectives: People with apparently well-defined demands may often have hidden or lately presented complaints or concerns, called hidden agenda, by the way syndrome or doorknob syndrome. Few studies that address the phenomenon in a clinical context discuss aspects or consequences on the care from a sociocultural perspective. A qualitative research on men's health conducted by the authors required to study the scientific literature related to hidden agenda in a descriptive and interpretative way. Methods: This state of question review encompasses articles that addressed hidden agenda (or similar expressions) in general or specialized, medical or multidisciplinary clinical settings, in Portuguese, English or Spanish, from 2000 to 2014 . Starting from 210 results from three international databases, we selected 39 publications, which were evaluated for objective and subjective characteristics. Results: Identifying the predominant subjects of the publications, we noticed that hidden agenda is the most specific term, being demanda oculta its best translation to Portuguese. Almost all studies related attention to hidden demands to better outcomes or results of care, although none of them evaluated those consequences in depth. Conclusions: The review makes it clear that hidden agenda is a problem-solving element to think about medical practice and an enhancer to the clinic, especially when looking for a comprehensive care and an effective dialogue between professional and user. Studies relating the phenomenon to sociocultural or healthcare service-related aspects are needed, as well as studies that include discussion about demand making, absent in the revised articles.

Como citar: Modesto AAD, Couto MT. Como se estuda o que não se diz: uma revisão sobre demanda oculta. Rev Bras Med Fam Comunidade. 2016;11(38):1-13. http://dx.doi.org/10.5712/rbmfc11(38)1250
Palavras-chave: Relação Médico-Paciente Comunicação Necessidades e Demandas de Serviços de Saúde Atenção Primária à Saúde

Keywords: Physician-Patient Relations Communication Health Services Needs and Demand Primary Health Care

Fonte de financiamento: declaram não haver. Parecer CEP: não se aplica. Conflito de interesses: declaram não haver. Procedência e revisão por pares: revisado por pares. Recebido em: 12/12/2015. Aprovado em: 04/05/2016. 


\section{Resumen}

Objetivos: Personas con demandas aparentemente bien definidas pueden frecuentemente tener quejas o inquietudes ocultas o tardíamente presentadas, llamadas en inglés de hidden agenda, by the way syndrome o doorknob syndrome ("síndrome del pomo de la puerta"). Pocos estudios en contexto clínico discuten sus aspectos o consecuencias para el cuidado desde un punto de vista sociocultural. Una investigación cualitativa realizada sobre salud masculina requirió estudiar la literatura científica relacionada con la hidden agenda de forma descriptiva e interpretativa. Métodos: Esta revisión, de tipo estado de la cuestión, abarcó artículos que abordaban la hidden agenda (o expresiones similares) en contextos clínicos generales o especializados, médicos o multidisciplinares, en portugués, inglés o español, de 2000 a 2014. Partiendo de 210 resultados de tres bases de datos internacionales, se seleccionaron 39 publicaciones, evaluadas cuanto a características objetivas y subjetivas. Resultados: Identificando temas predominantes en las pesquisas, observamos que hidden agenda es la expresión más específica, y demanda oculta, su mejor traducción. Casi todos los estudios relacionaban la atención a demandas ocultas a mejores resultados, aunque ninguno de ellos evaluaba estas consecuencias en profundidad. Conclusiones: La revisión deja claro que la demanda oculta es un elemento problematizador para pensar en la práctica médica y potenciador de la clínica, sobre todo al buscarse una atención integral y un diálogo efectivo entre profesional y usuario(a). Se necesitan estudios que relacionen el fenómeno con aspectos socioculturales y tecnoasistenciales, y que incluyan alguna discusión sobre construcción de la demanda, ausente de los artículos revisados.
Palabras clave:

Relaciones Médico-Paciente

Comunicación

Necesidades y Demandas

de Servicios de Salud

Atención Primaria de Salud

\section{Introdução}

Pessoas atendidas por demandas aparentemente bem delimitadas podem ter queixas ou preocupações ocultas ou tardiamente apresentadas, chamadas em inglês de hidden agenda. ${ }^{1,2}$ Barsky é considerado um pioneiro no assunto, graças a uma revisão em que discute as razões ocultas pelas quais pacientes procuram médicos. ${ }^{3} \mathrm{Nela}$, o autor demonstrava que "os aspectos não específicos do processo de cuidado médico têm sido geralmente consignados ao reino da idiossincrasia, intuição ou 'arte'”, e que era necessário "começar a descrevê-los com precisão e fazê-los quantificáveis, verificáveis e objetivos" (p. 497).

Beckman e Frankel, por outro lado, discordavam que a hidden agenda se tratasse apenas da retenção ou atraso de informação relevante pelo paciente, como se dependesse de sua vontade ou motivação. Focando na influência do comportamento médico na coleta de dados, eles identificaram que os profissionais interrompiam os pacientes durante seus relatos iniciais em quase $70 \%$ das vezes. Isso acontecia, em média, em 18 segundos de conversa, e quase nunca era dada nova oportunidade aos indivíduos para que completassem seu relato inicial - o que poderia levar à perda de informações relevantes. ${ }^{4}$

Diante do crescente reconhecimento da questão, muitos autores escreveram sobre hidden agenda em recortes específicos, como Hengstler et al., na clínica geral, ${ }^{5}$ e Ahmad e Ramsay, em dermatologia. ${ }^{6}$ Outros preferem o termo patient's agenda, ${ }^{7,8}$ embora essa expressão pareça significar o conjunto das questões que uma pessoa esperava que fossem abordadas em consulta, e não apenas as menos claras ou secundariamente expressadas. Também são conhecidos os apelidos by the way syndrome, referindo-se a "lembranças" no fim da consulta, e doorknob syndrome ("síndrome da maçaneta"), aludindo ao momento em que a pessoa pega a maçaneta da porta para deixar o consultório e expressa queixas ou preocupações novas - e, às vezes, mais importantes que as referidas até então..$^{9,10}$

Essa multiplicidade de termos pode indicar a necessidade de maior consolidação de um referencial, embora já esteja claro o impacto do fenômeno em qualquer contexto clínico - especialmente na Atenção Primária à Saúde (APS). Stewart et al. apontam que uma atenção médica que busca um campo comum com o paciente e responde mais completamente às suas necessidades únicas está associada a melhoras 
no estado de saúde (como menos preocupação) e na eficiência do cuidado (como menos exames e encaminhamentos). ${ }^{11}$ Starfield, por sua vez, afirma que integralidade de um serviço de APS depende do reconhecimento das necessidades dos pacientes por parte dos profissionais, mas estes frequentemente não conseguem reconhecer os problemas que aqueles expressam quando buscam atendimento - principalmente quando se tratam de questões de natureza psicológica ou psicossocial. ${ }^{12}$

A questão pode ser tratada no âmbito da psicologia e da psicanálise ${ }^{13,14}$ ou das habilidades de comunicação. ${ }^{15,16}$ Entretanto, poucos trabalhos que abordam o fenômeno da hidden agenda em contexto clínico discutem seus aspectos ou consequências sobre o cuidado do ponto de vista sociocultural.

Uma pesquisa qualitativa mais ampla realizada pelos autores vem estudando como os homens expressam demandas referentes à próstata e a questões de sexualidade (principalmente disfunção erétil) na APS, tentando entender as formas como essas demandas são expressas (ou não) pelos homens e acolhidas (ou não) pelos(as) médicos(as). Nesse contexto, mostrou-se fundamental revisar a produção científica relacionada à hidden agenda, explorando como tem sido estudada e nomeada, e em que recortes tem se mostrado útil.

A partir dos achados, esperamos demonstrar como essa noção pode ajudar a problematizar o contexto clínico, bem como fazer considerações sobre futuras pesquisas sobre o tema. Ressaltando que as diversas expressões encontradas em pesquisas preliminares não são necessariamente sinônimas, usaremos os termos no idioma original até estabelecermos suas diferenças, buscando contribuir à consolidação de um referencial e identificar a expressão mais adequada em português.

\section{Metodologia}

A revisão abarcou artigos que tratassem da hidden agenda (e termos similares) no contexto clínico geral ou especializado, médico ou multiprofissional, em português, inglês e espanhol, no período de 2000 a 2014. A metodologia utilizada e o caráter simultaneamente descritivo e interpretativo permitem definir esta revisão como do tipo estado da questão, tendo a mesma finalidade apontada por Nóbrega-Therrien e Therrien ${ }^{17}$ de "levar o pesquisador a registrar, a partir de um rigoroso levantamento bibliográfico, como se encontra o tema ou o objeto de sua investigação no estado atual da ciência ao seu alcance" (p. 7). Um exemplo de revisão desse tipo é a pesquisa de Cavalcante Neto et al. sobre o interesse dos estudantes pela Medicina de Família. ${ }^{18}$

Buscamos as combinações de termos listados na Tabela 1 nos portais PubMed, Biblioteca Virtual de Saúde (BVS) e SciELO (que abrangem bases de dados internacionais como MEDLINE, LILACS e Cochrane) no primeiro trimestre de 2015. As expressões deveriam constar do título, assunto ou resumo das publicações. Como hidden agenda e patient's agenda pareciam os termos mais usados na literatura científica anglófona, fizemos combinações com agenda, demand e demanda esperando revelar sinônimos ou textos em português ou espanhol. As quatro últimas combinações pesquisadas, por sua vez, foram inspiradas por um relato de caso encontrado em pesquisas preliminares que destacava a importância de "escutar a demanda subjacente à queixa enunciada"13 (p. 329). A Tabela 1 mostra também as combinações de termos e os resultados que cada uma gerou em cada portal (descontados resultados duplicados em uma mesma pesquisa). 
Tabela 1. Resultados por combinação de termos e portais.

\begin{tabular}{lcccc}
\hline & \multicolumn{3}{c}{ Portal } \\
\cline { 2 - 5 } Termo & ScieLO & BVS & PubMed & Total \\
\hline "demanda do paciente" OR "demanda del paciente" & 16 & 54 & 49 & 119 \\
OR "patient's demand" & 3 & 30 & 57 & 90 \\
"agenda oculta" OR "hidden agenda" & 0 & 24 & 31 & 55 \\
"agenda do paciente" OR "agenda del paciente" & 0 & 21 & 27 & 48 \\
OR "patient's agenda" & 0 & 4 & 5 & 9 \\
"demanda latente" OR "latent demand" & 2 & 2 & 0 & 4 \\
"demanda subjacente" OR "underlying demand" & 0 & 1 & 0 & 1 \\
"demanda oculta" OR "hidden demand" & 0 & 0 & 0 & 0 \\
"agenda subjacente" OR "underlying agenda" & 21 & 136 & 169 & 326 \\
Total & & &
\end{tabular}

Cruzando os resultados dos três portais e desprezando repetições, chegamos a uma lista com 210 títulos. Eliminando resultados repetidos, anteriores a 2000 ou em idioma que não inglês, português ou espanhol, passamos a 117 resultados. Excluímos textos com versão online indisponível (11), além de publicações que não fossem artigos (como teses, comentários e editoriais), artigos em que a expressão era citada de forma superficial, sem maiores considerações, e pesquisas que se situavam fora do contexto clínico (levando a 67 exclusões). A partir desses critérios, 39 artigos foram selecionados.

Os trabalhos selecionados foram identificados quanto ao ano e idioma de publicação, país de realização, tipo de estudo e área do saber (medicina, enfermagem, psicologia etc.). Na sequência, avaliamos: 1) se a pesquisa era conduzida da perspectiva de usuários, profissionais ou ambos; 2) se envolvia homens, mulheres ou ambos; 3) como a noção se caracterizava e era abordada no contexto investigado; 4) que temas eram abordados; e 5) se contemplava desfechos ou desdobramentos do atendimento.

A análise qualitativa dos cinco aspectos destacados percorreu os seguintes passos: 1) Leitura flutuante dos artigos com o objetivo de apreender o conteúdo geral dos artigos segundo os aspectos em análise; 2) Identificação e agrupamento dos argumentos dos autores (relativos aos três últimos aspectos de análise) em núcleos de sentido; 3) Classificação e interpretação dos núcleos de sentidos em eixos de análise mais abrangentes; 4) Sínteses interpretativas a partir dos núcleos de sentido mais recorrentes nos estudos. Todos esses passos seguiram a proposta de análise qualitativa de Minayo. ${ }^{19}$

\section{Resultados}

A distribuição dos textos por ano de publicação variou entre zero e seis (na média, quase três por ano). Vinte e três estudos (quase $60 \%$ do total) foram realizados na Europa, sendo que em nove deles o Reino Unido estava envolvido. Três pesquisas foram realizadas em mais de um país. Os Estados Unidos também tiveram grande participação, com nove estudos ( $23 \%$ do total). A América do Sul sediou apenas quatro, sendo dois deles no Brasil. Outras características são mostradas na Tabela 2 e na Figura 1.

Os termos utilizados nas publicações selecionadas estão na Tabela 3, acompanhados dos temas mais associados a cada noção - o que discutiremos a seguir. 
Tabela 2. Características dos artigos selecionados.

\begin{tabular}{|c|c|c|c|}
\hline \multicolumn{2}{|c|}{ Tipo de estudo } & \multicolumn{2}{|c|}{ Idioma do artigo } \\
\hline Quantitativo & 16 & Inglês & 34 \\
\hline Qualitativo & 8 & Espanhol & 3 \\
\hline Revisão & 5 & Português & 2 \\
\hline Qualitativo e quantitativo & 4 & \multicolumn{2}{|c|}{ Perspectiva do estudo } \\
\hline Relato de caso & 4 & Profissionais & 17 \\
\hline \multicolumn{2}{|c|}{ Sexo dos(as) participantes } & Ambos & 13 \\
\hline Homens e mulheres & $30^{*}$ & Usuários(as) & 9 \\
\hline Não consideravam & $5^{\star *}$ & \multicolumn{2}{|c|}{ Desfechos ou desdobramentos } \\
\hline Apenas mulheres & 3 & Contemplavam & 34 \\
\hline Apenas homens & 1 & Não faziam considerações & 5 \\
\hline
\end{tabular}

${ }^{*}$ Houve diferença entre os grupos de participantes em três estudos (e.g., profissionais de ambos os sexos e usuárias mulheres); ${ }^{* \star}$ Dois ensaios, duas revisões e uma pesquisa qualitativa.

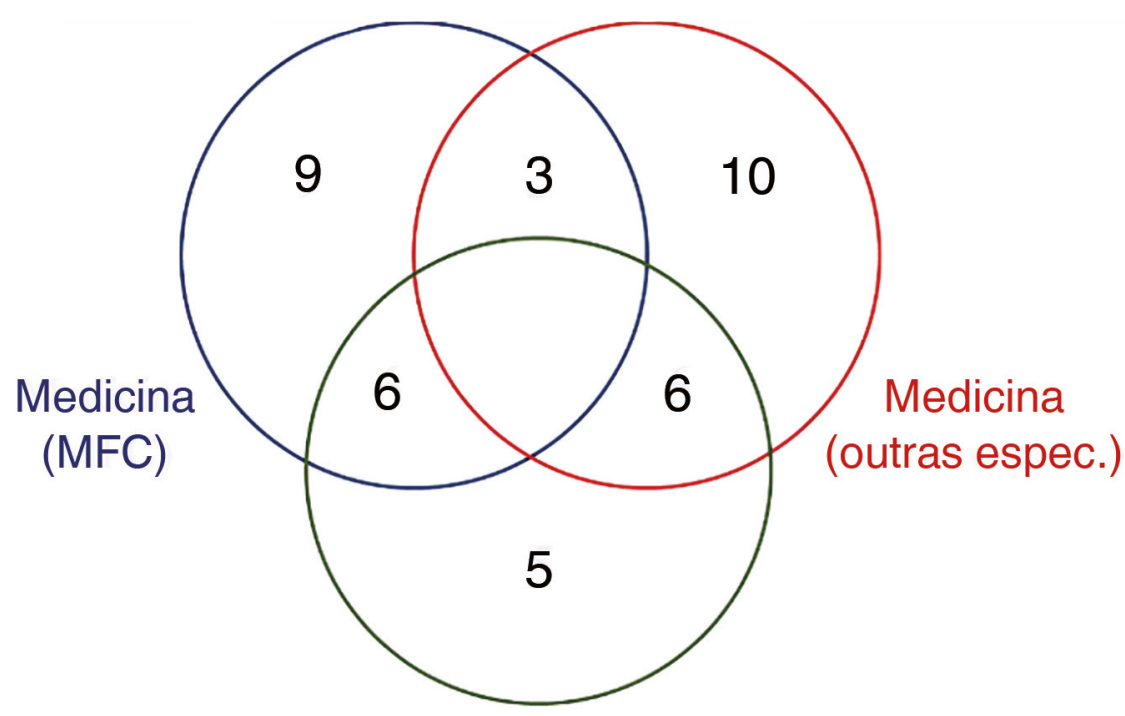

\section{Outras áreas}

Figura 1. Publicações selecionadas por área do saber.

MFC: Medicina de Família e Comunidade (e equivalentes estrangeiros); Outras espec.: outras especialidades médicas; Outras áreas: enfermagem, farmácia, odontologia, psicologia/psicanálise.

Tabela 3. Termos utilizados e temas predominantes nas publicações selecionadas.

\begin{tabular}{lcl}
\hline Termo & Número & Temas predominantes \\
\hline Patient's agenda & 17 & $\begin{array}{l}\text { Registro médico informatizado; barreiras comunicacionais em condições específicas de saúde; } \\
\text { comunicação em atendimentos em geral; intervenções para qualificar o atendimento médico }\end{array}$ \\
Patient's demand & 8 & $\begin{array}{l}\text { Demandas específicas (normalmente claras) e tomada de decisão } \\
\text { Demandas não percebidas pelos profissionais, maior relação entre identificação e desfechos, } \\
\text { Hidden agenda }\end{array}$ \\
$\begin{array}{lll}\text { Demanda del paciente } \\
\text { memaior circunscrição à clínica }\end{array}$ \\
Latent demand & 4 & Saúde mental \\
\hline
\end{tabular}




\section{Discussão}

O processo de seleção dos resultados merece alguns comentários, por demonstrar a polissemia dos termos pesquisados. Demanda do/del paciente e patient's demand surgiram frequentemente em ensaios clínicos de analgesia pós-operatória, ${ }^{20,21}$ enquanto hidden agenda e agenda oculta se referiam a técnicas de moderação de grupos, ${ }^{22}$ intenções não declaradas de governos ou grupos influentes ${ }^{23}$ ou problemas populacionais não contemplados por políticas públicas ${ }^{24}$ - caso também do único resultado com o termo demanda oculta ${ }^{25}$ excluído por colocar-se em âmbito mais epidemiológico do que clínico. Muitos resultados iniciais com latent demand foram excluídos por se referirem a demandas populacionais por questões não propriamente clínicas (como transporte ${ }^{26}$ e água potáve ${ }^{27}$ ), enquanto outros, embora tratassem de temas interessantes à clínica, tinham abordagens muito mais próximas da avaliação de mercado ou do uso de recursos (como a demanda latente por contracepção pericoital ${ }^{28}$ ou imunoglobulina ${ }^{29}$ em regiões específicas).

A ocorrência de estudos em diferentes áreas médicas, não médicas e multidisciplinares mostra que o assunto é de interesse geral da saúde, e o destaque da Medicina de Família e Comunidade e seus equivalentes estrangeiros entre as pesquisas corrobora a APS como um campo importante para a problematização do fenômeno.

A abordagem predominante nas pesquisas empíricas foi quantitativa, muitas vezes envolvendo gravações de consultas em áudio ou vídeo, questionários ou avaliação de intervenções educacionais. $A$ perspectiva dos profissionais foi considerada em mais de três quartos dos estudos, sendo associada à dos usuários em cerca de um terço desses. O predomínio de produções no hemisfério norte e em língua inglesa e a presença de apenas dois estudos brasileiros mostram uma lacuna para a pesquisa sul-americana e nacional, cujo preenchimento poderia contribuir na validação do conceito e no aporte de aspectos mais específicos ao nosso contexto.

As publicações selecionadas estão listadas conforme o termo utilizado na Tabela 4. Doze expressões pesquisadas não constavam de nenhum dos resultados selecionados, incluindo demanda oculta e agenda oculta. Os cinco textos em português ou espanhol usavam as expressões demanda do/de/ paciente ou a versão patient's demand no resumo.

As publicações que usavam a expressão patient's agenda abordavam quatro temas principais. $\mathrm{O}$ primeiro grupo incluía pesquisas sobre registro médico informatizado e seu impacto sobre o entendimento das demandas do sujeito - a patient's agenda. Snoek et al. consideram que a informática ajudou na identificação das necessidades individuais de diabéticos em seguimento, ${ }^{30,31}$ enquanto Rhodes et al. criticam o uso de um checklist computadorizado para o mesmo tipo de atendimento. ${ }^{32}$ Impactos negativos do uso de prontuário eletrônico também foram apontados por Makoul et al. ${ }^{33}$

O segundo grupo de artigos contemplava barreiras comunicacionais em condições específicas de saúde: fadiga; ${ }^{34}$ dispepsia; ${ }^{35}$ câncer de mama; ${ }^{36}$ multimorbidade e polifarmácia; ${ }^{37}$ risco cardiovascular em mulheres ${ }^{38}$ e cuidados intensivos. ${ }^{39}$ Tomando a obesidade como problema, Sussman et al. investigaram fatores que influenciam a decisão médica em oferecer aconselhamento preventivo em consultas de APS, demonstrando que a demanda do paciente foi o fator mais importante para os entrevistados na decisão em investir tempo em aconselhamento ${ }^{40}$ - o que o aproxima dos estudos de patient's demand, como veremos a seguir. 
Tabela 4. Publicações selecionadas e tipos de estudo conforme termos utilizados.

\begin{tabular}{|c|c|c|c|}
\hline \multicolumn{2}{|c|}{ Patient's agenda } & \multicolumn{2}{|c|}{ Patient's demand } \\
\hline Makoul et al., 2001 & Qualitativo e quantitativo & Kasje et al., 2002 & Qualitativo \\
\hline Meeuwesen et al., 2002 & Quantitativo & Jacquemyn et al., 2003 & Quantitativo \\
\hline Sepucha et al., 2002 & Quantitativo & Bremberg e Nilstun, 2005 & Quantitativo \\
\hline Vegni et al., 2002 & Quantitativo & Cahana et al., 2008 & Quantitativo \\
\hline Jones, 2003 & Revisão & Fernández, 2008 & Ensaio \\
\hline Dyche e Swiderski, 2005 & Quantitativo & Hunt et al., 2011 & Qualitativo e quantitativo \\
\hline Huffman, 2005 & Revisão & Aulakh e Melsen, 2011 & Relato de caso \\
\hline Frich et al., 2006 & Qualitativo & Kitzinger et al., 2012 & Quantitativo \\
\hline Rhodes et al., 2006 & Qualitativo & \multicolumn{2}{|c|}{ Hidden agenda } \\
\hline Sussman et al., 2006 & Qualitativo e quantitativo & Hengstler et al., 2002 & Revisão \\
\hline Rodondi et al., 2009 & Quantitativo & McLeod, 2003 & Ensaio \\
\hline Bower et al., 2011 & Qualitativo & Gross et al., 2008 & Quantitativo \\
\hline Snoek et al., 2011 & Quantitativo & Ahmad e Ramsay, 2009 & Qualitativo e quantitativo \\
\hline Altiner et al., 2012 & Quantitativo & Fallon et al., 2012 & Relato de caso \\
\hline Snoek et al., 2012 & Quantitativo & Tempark e Shwayder, 2012 & Relato de caso \\
\hline Hazzard et al., 2013 & Revisão & \multicolumn{2}{|c|}{ Demanda do paciente } \\
\hline Weiss et al., 2013 & Quantitativo & leto e Cunha, 2007 & Relato de caso \\
\hline \multicolumn{2}{|c|}{ Demanda del paciente } & Costa et al., 2009 & Revisão \\
\hline Nakai et al., 2009 & Quantitativo & \multicolumn{2}{|c|}{ Latent demand } \\
\hline Gallo et al., 2012 & Qualitativo & Burkey et al., 2006 & Quantitativo \\
\hline Desplenter et al., 2013 & Qualitativo & Packel et al., 2012 & Qualitativo \\
\hline Miranda Hiriart e Saffie Gatica, 2014 & Qualitativo & & \\
\hline
\end{tabular}

O terceiro grupo tratava da comunicação em atendimentos em geral. Dyche e Swiderski estudaram a compreensão médica sobre as demandas dos pacientes, ${ }^{41}$ enquanto Rodondi et al. estudaram o conteúdo e as respostas médicas à by the way syndrome, registrando um desencontro entre o conteúdo da síndrome (normalmente biopsicossocial ou psicossocial) e as respostas médicas (normalmente biomédicas $)^{10}$ - condizendo com o que apontou Starfield. ${ }^{12}$ Huffman fez uma revisão sobre parceria no cuidado, ${ }^{42}$ recomendando reservar tempo para determinar o que é importante para o paciente e seu cuidador, e Weiss et al. observaram similaridades entre médicos de família, enfermeiras e farmacêuticos prescritores britânicos no quão pouco seus pacientes completavam seu relato inicial ( $20 \%$ das consultas) e quão rapidamente eram redirecionados (22 a 28 segundos, em média). ${ }^{43}$

O último grupo de publicações estudou intervenções para qualificar o atendimento médico, por meio do treinamento em habilidades de comunicação e medicina centrada na pessoa, com resultados promissores. ${ }^{44,45}$

Oito publicações usaram a expressão patient's demand. Kasje et al. demonstraram que médicos holandeses tendem a atender a demanda do paciente por medicações reembolsáveis, ${ }^{46}$ enquanto Jacquemyn et al. mostraram que a demanda da gestante por cesariana era um fator pouco importante na escolha dessa via de parto por obstetras flamencos. ${ }^{47}$ Bremberg e Nilstun compararam as escolhas e justificativas de médicos de família e comunidade, oncologistas e público leigo em situações hipotéticas em que alguém reluta em aceitar uma intervenção médica ou demanda ações medicamente injustificáveis, 
encontrando discordância entre profissionais e público leigo. ${ }^{48}$ Cahana et al. compararam a atitude de quatro categorias profissionais a respeito da transfusão de sangue a Testemunhas de Jeová que tivessem recusado antecipadamente esse procedimento. Questionários foram aplicados antes e depois de uma aula sobre bioética. ${ }^{49}$ Patient's demand também se referia a demandas específicas de pacientes e sua influência na tomada de decisão em um ensaio sobre psicoterapia de apoio em APS ${ }^{50}$ e em duas publicações de odontologia: uma pesquisa sobre sedação intravenosa a pacientes ansiosos ${ }^{51}$ e um relato de caso em que atender cegamente à demanda de uma paciente teria levado a um tratamento insatisfatório e com pior prognóstico. ${ }^{52}$ Kitzinger et al., por fim, recomendam que cirurgiões atentem à demanda por cirurgia plástica corporal de pacientes submetidos a cirurgia bariátrica. ${ }^{53}$

Quase todos os estudos que usavam a expressão demanda do/de/ paciente tratavam de saúde mental. Para leto e Cunha, a demanda subjacente à queixa é um aspecto a avaliar no atendimento fonoaudiológico, ${ }^{13}$ enquanto para Desplenter et al. profissionais fornecem informações sobre antidepressivos a pacientes internados de acordo com a demanda destes. ${ }^{54}$ Costa et al. destacam que identificar a demanda do paciente é parte da avaliação psicológica de obesos. ${ }^{55}$ Dois estudos debatem o sofrimento psíquico de pacientes policonsultantes e dos profissionais que os atendem. ${ }^{14,56}$ Ambos destacam o desencontro das expectativas de uns e outros e mostram que o vínculo é uma estratégia mais eficaz no cuidado dessas pessoas que o afastamento e a medicalização que as unidades comumente lhes oferecem. Finalmente, o estudo de Nakai et al. apontou que a demanda do paciente foi o fator que mais motivou farmacêuticos a oferecerem pacotes de dose única diária de medicação a idosos ${ }^{57}$ - diferenciando-se dos demais e aproximando-se ao grupo da patient's demand.

Os estudos que usavam a expressão latent demand se colocavam no limite entre a identificação de necessidades de saúde, demandas ao serviço ou mercados potenciais, mas mostravam algum desdobramento para a clínica. Burkey et al. revisaram dezenas de milhares de prontuários em busca de candidatos ao BAHA (Bone-Anchored Hearing Aid, aparelho auditivo de ancoragem óssea), demostrando superficialmente a importância dessa busca ativa. ${ }^{58}$ Packel et al., por sua vez, identificaram uma demanda latente por testagem de doenças sexualmente transmissíveis enquanto estudavam o pagamento de bônus por resultado negativo como estratégia para evitar a infecção. ${ }^{59}$

As publicações que usavam a expressão hidden agenda também abordavam demandas não percebidas pelos profissionais, diferenciando-se das anteriores pela maior relação que estabeleciam entre o reconhecimento dessas demandas e os desfechos do atendimento, pelo entendimento consequente de que identificá-las é um elemento fundamental da consulta, e pela maior circunscrição de seus objetos à clínica. Tudo isso a coloca como a expressão mais específica dentre as pesquisadas.

Hengstler et al. revisaram recomendações de rastreamento em adultos, observando que pacientes frequentemente usam o check-up como razão para consulta para levantar um problema específico que os incomoda e aconselhando os médicos a abordar possíveis hidden agendas nessas pessoas. ${ }^{5}$ Gross et al. abordaram a percepção de hidden agenda entre médicos e médicas atendendo pessoas do mesmo sexo ou diferente. ${ }^{60}$ Ahmad e Ramsay exploraram medos e expectativas de pacientes dermatológicos por meio de questionários a profissionais e usuários, sugerindo o uso desses instrumentos para otimizar a consulta e melhorar o cuidado. ${ }^{6}$ Fallon et al. estudaram a hidden agenda de jovens cuidadores ou usuários de serviços de saúde mental, sugerindo incluir esses jovens no planejamento dos serviços de saúde e 
educação. ${ }^{61}$ Tempark e Shwayder relataram dois atendimentos de crianças de famílias chinesas cujos pais exigiam a remoção de manchas em seus rostos, a despeito da benignidade das lesões e da futura cicatriz. A insistência foi posteriormente relacionada a uma crença chinesa na leitura da sorte pela posição de manchas no corpo e no rosto. ${ }^{62} \mathrm{O}$ ensaio reflexivo de McLeod se diferenciava um pouco dos demais, voltando-se a questões subconscientes dos profissionais que podem influenciar o atendimento. ${ }^{63}$

Quase todos os estudos relacionavam a atenção à hidden agenda, patient's agenda etc. a melhores desfechos ou desdobramentos do atendimento, como maior satisfação do(a) usuário(a) e profissional, maior adesão ao tratamento e superação de barreiras diagnósticas e comunicacionais. Alguns chegavam a advertir sobre consequências negativas de não atentar a essas questões, como cronificação de doenças, ${ }^{34}$ atraso no diagnóstico, ${ }^{38}$ prejuízo ao processo terapêutico, ${ }^{6}$ excesso de consultas e sobrecarga assistencial. ${ }^{14,56}$

Embora nenhum estudo avaliasse essas consequências de forma aprofundada, a revisão deixa claro que "revelar a hidden agenda é crucial para fazer um diagnóstico biopsicossocial integral, permitindo ao médico oferecer tratamento apropriado, e está relacionado às habilidades de comunicação de escutar e interpretar pistas não verbais" (p. 126). ${ }^{60}$ Dyche e Swiderski ${ }^{41}$ afirmam que "quando os médicos entendem as preocupações dos pacientes, há uma melhora na satisfação e adesão do paciente" (p. 270), fazendo coro com Stewart et al., ${ }^{11}$ enquanto Weiss et al. ${ }^{43}$ encorajam os(as) profissionais a usar mais questões abertas e perguntar por outras demandas várias vezes ao longo da consulta, esperando que isso resulte em maior expressão de preocupações e melhores resultados. Como disse Barsky, ${ }^{3}$ "um clínico poderia investigar a causa da visita tão sistematicamente quanto ele ou ela investiga a causa da febre, fadiga ou perda de peso de um paciente" (p. 497).

\section{Considerações finais}

A restrição a 15 anos de produção e 3 mecanismos de busca são duas limitações da nossa revisão, mas o número de resultados iniciais por portal $(26,136$ e 169) e da lista unificada (210 títulos) mostra que alcançamos um panorama satisfatório da literatura contemporânea sobre o assunto, e sugere que a inclusão de outras bases de dados não acrescentaria informações essenciais. Outra limitação foi a pesquisa por expressões fechadas, que pode ter deixado de fora publicações que tratavam do assunto usando outras palavras. Finalmente, a combinação de critérios objetivos e subjetivos na seleção das publicações pode parecer uma limitação a leitores acostumados a revisões sistemáticas ou meta-análises, mas é coerente à pesquisa qualitativa que a inspirou, à revisão do tipo estado da questão, e ao próprio assunto estudado. Além disso, o processo de inclusão e exclusão nos permite afirmar que todos os artigos selecionados ofereciam algum aporte a futuras pesquisas sobre o fenômeno da hidden agenda em contexto clínico impressão que tentamos transmitir por meio dos comentários sobre os artigos excluídos.

A análise dos temas relacionados às diferentes expressões pesquisadas mostrou que hidden agenda é a mais específica ao fenômeno em questão. Agenda em inglês se refere a pauta ou lista de assuntos a discutir (significados menos comuns para agenda em português), enquanto demand significa exigência ou condição, não correspondendo exatamente ao que chamamos de demanda. ${ }^{64}$ Assim, mesmo não tendo sido usada em nenhum artigo selecionado (sinal da pouca produção lusófona sobre o tema), demanda oculta parece a melhor forma de chamar as queixas ou preocupações tardias ou mais dificilmente expressadas. 
Há uma literatura robusta mostrando que os profissionais de saúde não investigam demandas ocultas, sugerindo motivos para tanto e ensinando-os a fazê-lo, mas parece haver pouca discussão sobre por que isso ocorre. Nossa revisão mostra que a demanda oculta é um elemento problematizador para pensar a prática médica e potencializador para a clínica, especialmente considerando-se a busca por um cuidado integral e um efetivo diálogo entre profissional e usuário. Merece, portanto, a realização de estudos que tentem articular o fenômeno a aspectos socioculturais, como gênero, ${ }^{65}$ ou tecnoassistenciais, como prevenção quaternária ${ }^{66}$ e gestão da clínica. ${ }^{67}$

Outro aspecto a ser considerado em pesquisas futuras é a ausência de discussão sobre a construção da demanda nos artigos revisados, levando a crer que os autores entendem que as demandas identificadas nas pesquisas eram necessidades prontas, objetiváveis e conhecidas pelo sujeito, que aguardavam apenas sua decisão de expressá-las, a oportunidade de surgir em consulta ou o escrutínio médico - condizendo com os entendimentos pioneiros de Barsky e de Beckman e Frankel. Essa visão, no entanto, desconsidera os profissionais e os próprios pesquisadores como geradores de demandas; que o próprio serviço seja um "contexto instaurador de necessidades";68 que a demanda possa ser construída ao longo do processo terapêutico; ${ }^{69}$ e que o vínculo possa cumprir um papel importante na comunicação. Ter isso em mente pode nos ajudar a entender por que algumas questões permanecem não ditas mesmo ao pegar a maçaneta da porta.

\section{Referências}

1. Menahem S. Teaching students of medicine to listen: the missed diagnosis from a hidden agenda. J R Soc Med. 1987;80(6):343-6.

2. Silverman J. Hidden agendas and how to uncover them. Medicine. 2005;33(2):27-9. DOI: http://dx.doi.org/10.1383/ medc.33.2.27.58375

3. Barsky AJ 3rd. Hidden reasons some patients visit doctors. Ann Intern Med. 1981;94(4 pt 1):492-8. DOI: http://dx.doi. org/10.7326/0003-4819-94-4-492

4. Beckman HB, Frankel RM. The effect of physician behavior on the collection of data. Ann Intern Med. 1984;101(5):692-6. DOI: http://dx.doi.org/10.7326/0003-4819-101-5-692

5. Hengstler P, Battegay E, Cornuz J, Bucher H, Battegay M. Evidence for prevention and screening: recommendations in adults. Swiss Med Wkly. 2002;132(27-28):363-73. PMID: 12428190

6. Ahmad K, Ramsay B. Patients' fears and expectations: exploring the hidden agenda in our consultation. Arch Dermatol. 2009;145(6):722-3. PMID: 19528436 DOI: http://dx.doi.org/10.1001/archdermatol.2009.102

7. Marvel MK, Epstein RM, Flowers K, Beckman HB. Soliciting the patient's agenda: have we improved? JAMA. 1999;281(3):283-7. DOI: http://dx.doi.org/10.1001/jama.281.3.283

8. Barry CA, Bradley CP, Britten N, Stevenson FA, Barber N. Patients' unvoiced agendas in general practice consultations: qualitative study. BMJ. 2000;320(7244):1246-50. DOI: http://dx.doi.org/10.1136/bmj.320.7244.1246

9. Jackson G. "Oh ... by the way ...": doorknob syndrome. Int J Clin Pract. 2005;59(8):869. DOI: http://dx.doi.org/10.1111/j.13685031.2005.0599a.x

10. Rodondi PY, Maillefer J, Suardi F, Rodondi N, Cornuz J, Vannotti M. Physician response to "by-the-way" syndrome in primary care. J Gen Intern Med. 2009;24(6):739-41. DOI: http://dx.doi.org/10.1007/s11606-009-0980-2

11. Stewart M, Brown JB, Donner A, McWhinney IR, Oates J, Weston WW, et al. The impact of patient-centered care on outcomes. J Fam Pract. 2000;49(9):796-804. PMID:11032203

12. Starfield B. Atenção Primária: equilíbrio entre necessidades de saúde, serviços e tecnologia. Brasília: UNESCO/Ministério da Saúde; 2002. p.726. 
13. leto V, Cunha MC. Queixa, demanda e desejo na clínica fonoaudiológica: um estudo de caso clínico. Rev Soc Bras Fonoaudiol. 2007;12(4):329-34. DOI: http://dx.doi.org/10.1590/S1516-80342007000400013

14. Echeverri Gallo C, Alvarez Restrepo A, Londono Bernal LV. Aspectos subjetivos implicados en la demanda de consulta médica frecuente. Psicol Caribe. 2012;29(2):511-37.

15. Ribeiro MMF, Amaral CFS. Medicina centrada no paciente e ensino médico: a importância do cuidado com a pessoa e o poder médico. Rev Bras Educ Med. 2008;32(1):90-7. DOI: http://dx.doi.org/10.1590/S0100-55022008000100012

16. Leite AJM, Caparara A, Coelho Filho JM, orgs. Habilidades de comunicação com pacientes e famílias. São Paulo: Sarvier; 2007.

17. Nóbrega-Therrien SM, Therrien J. Trabalhos científicos e o estado da questão: reflexões teórico-metodológicas. Estud Aval Educ. 2004;15(30):5-16. DOI: http://dx.doi.org/10.18222/eae153020042148

18. Cavalcante Neto PG, Lira GV, Miranda AS. Interesse dos estudantes pela medicina de família: estado da questão e agenda de pesquisa. Rev Bras Educ Med. 2009;33(2):198-204. DOI: http://dx.doi.org/10.1590/S0100-55022009000200006

19. Minayo MCS. O desafio do conhecimento: pesquisa qualitativa em saúde. 2a ed. São Paulo/Rio de Janeiro: Hucitec/ Abrasco; 1993.

20. Pernia A, Torres LM, Calderón E. Tratamiento del dolor postoperatorio mediante analgesia intravenosa controlada por el paciente (P C A i. v.). Comparación entre propacetamol y metamizol. Rev Soc Esp Dolor. 2000;7(6):354-60.

21. Oliveira RM, Tenório SB, Tanaka PP, Precoma D. Controle da dor por bloqueio peridural e incidência de disritmias cardíacas no pós-operatório de procedimentos cirúrgicos torácicos e abdominais altos: estudo comparativo. Rev Bras Anestesiol. 2012;62(1):14-8. DOI: http://dx.doi.org/10.1590/S0034-70942012000100003

22. Guix J. Analizando los "porqués”: Ios grupos focales. Rev Calid Asist. 2003;18(7):598-602. DOI: http://dx.doi.org/10.1016/ S1134-282X(03)77644-1

23. Penson DF. The hidden agenda in the release of the Medicare physician reimbursement data. Urology. 2014;84(3):501-2. DOI: http://dx.doi.org/10.1016/j.urology.2014.06.001

24. Tesfay F. AIDS/HIV pandemic: the hidden agenda. J Natl Med Assoc. 2001;93(11):446-8. PMID: 11730119

25. Santos-Padrón H, Martínez-Calvo S, Martínez-López MC, Álvarez-Malpica IL. La demanda potencial y la oferta de servicios de salud para las enfermedades catastróficas en México. Rev Gerenc Polit Salud. 2011;10(21):33-47.

26. Diana M, Song T, Wittkowski KM. Studying travel-related individual assessments and desires by combining hierarchically structured ordinal variables. Transportation (Amst). 2009;36(2):187-206. DOI: http://dx.doi.org/10.1007/s11116-009-9186-z

27.Vásquez WF, Mozumder P, Hernández-Arce J, Berrens RP. Willingness to pay for safe drinking water: Evidence from Parral, Mexico. J Environ Manage. 2009;90(11):3391-400. DOI: http://dx.doi.org/10.1016/j.jenvman.2009.05.009

28. Cover JK, Drake JK, Kyamwanga IT, Turyakira E, Dargan T, Kumakech E, et al. Consumer perspectives on a pericoital contraceptive pill in India and Uganda. Int Perspect Sex Reprod Health. 2013;39(4):195-204. DOI: http://dx.doi. org/10.1363/3919513

29. Stonebraker JS, Farrugia A, Gathmann B; ESID Registry Working Party, Orange JS. Modeling primary immunodeficiency disease epidemiology and its treatment to estimate latent therapeutic demand for immunoglobulin. J Clin Immunol. 2014;34(2):233-44. DOI: http://dx.doi.org/10.1007/s10875-013-9975-1. DOI: http://dx.doi.org/10.1007/s10875-013-9975-1

30. Snoek FJ, Kersch NY, Eldrup E, Harman-Boehm I, Hermanns N, Kokoszka A, et al. Monitoring of Individual Needs in Diabetes (MIND): baseline data from the Cross-National Diabetes Attitudes, Wishes, and Needs (DAWN) MIND study. Diabetes Care. 2011;34(3):601-3. DOI: http://dx.doi.org/10.2337/dc10-1552

31. Snoek FJ, Kersch NY, Eldrup E, Harman-Boehm I, Hermanns N, Kokoszka A, et al. Monitoring of Individual Needs in Diabetes (MIND)-2: follow-up data from the cross-national Diabetes Attitudes, Wishes, and Needs (DAWN) MIND study. Diabetes Care. 2012;35(11):2128-32. DOI: http://dx.doi.org/10.2337/dc11-1326

32. Rhodes P, Langdon M, Rowley E, Wright J, Small N. What does the use of a computerized checklist mean for patientcentered care? The example of a routine diabetes review. Qual Health Res. 2006;16(3):353-76. DOI: http://dx. doi. org/10.1177/1049732305282396

33. Makoul G, Curry RH, Tang PC. The use of electronic medical records: communication patterns in outpatient encounters. J Am Med Inform Assoc. 2001;8(6):610-5. DOI: http://dx.doi.org/10.1136/jamia.2001.0080610 
34. Meeuwesen L, Bensing J, van den Brink-Muinen A. Communicating fatigue in general practice and the role of gender. Patient Educ Couns. 2002;48(3):233-42. DOI: http://dx.doi.org/10.1016/S0738-3991(02)00176-3

35. Jones MP. Evaluation and treatment of dyspepsia. Postgrad Med J. 2003;79(927):25-9. DOI: http://dx.doi.org/10.1136/ pmj.79.927.25

36. Sepucha KR, Belkora JK, Mutchnick S, Esserman LJ. Consultation planning to help breast cancer patients prepare for medical consultations: effect on communication and satisfaction for patients and physicians. J Clin Oncol. 2002;20(11):2695700. DOI: http://dx.doi.org/10.1200/JCO.2002.10.068

37. Bower P, Macdonald W, Harkness E, Gask L, Kendrick T, Valderas JM, et al. Multimorbidity, service organization and clinical decision making in primary care: a qualitative study. Fam Pract. 2011;28(5):579-87. DOI: http://dx.doi.org/10.1093/ fampra/cmr018

38. Frich JC, Malterud K, Fugelli P. Women at risk of coronary heart disease experience barriers to diagnosis and treatment: a qualitative interview study. Scand J Prim Health Care.2006;24(1):38-43. DOI: http://dx.doi.org/10.1080/02813430500504305

39. Hazzard A, Harris W, Howell D. Taking care: practice and philosophy of communication in a critical care follow-up clinic. Intensive Crit Care Nurs. 2013;29(3):158-65. DOI: http://dx.doi.org/10.1016/j.iccn.2013.01.003

40. Sussman AL, Williams RL, Leverence R, Gloyd PW Jr, Crabtree BF. The art and complexity of primary care clinicians' preventive counseling decisions: obesity as a case study. Ann Fam Med.2006;4(4):327-33. DOI: http://dx.doi.org/10.1370/ afm.566

41. Dyche L, Swiderski D. The effect of physician solicitation approaches on ability to identify patient concerns. J Gen Intern Med. 2005;20(3):267-70. DOI: http://dx.doi.org/10.1111/j.1525-1497.2005.40266.x

42. Huffman MH. Compliance, health outcomes, and partnering in PPS: acknowledging the patient's agenda. Home Healthc Nurse. 2005;23(1):23-8.

43. Weiss MC, Platt J, Riley R, Taylor G, Horrocks S, Taylor A. Solicitations in GP, nurse and pharmacist prescriber consultations: an observational study. Fam Pract. 2013;30(6):712-8. DOI: http://dx.doi.org/10.1093/fampra/cmt042

44. Vegni E, Martinoli M, Moja EA. Improving patient-centred medicine: a preliminary experience for teaching communication skills to Italian general practitioners. Educ Health (Abingdon). 2002;15(1):51-7. DOI: http://dx.doi. org/10.1080/13576280110107314

45. Altiner A, Schäfer I, Mellert C, Löffler C, Mortsiefer A, Ernst A, et al. Activating GENeral practitioners dialogue with patients on their Agenda (MultiCare AGENDA) study protocol for a cluster randomized controlled trial. BMC Fam Pract. 2012;13:118. DOI: http://dx.doi.org/10.1186/1471-2296-13-118

46. Kasje WN, Timmer JW, Boendermaker PM, Haaijer-Ruskamp FM. Dutch GPs' perceptions: the influence of out-of-pocket costs on prescribing. Soc Sci Med. 2002;55(9):1571-8. DOI: http://dx.doi.org/10.1016/S0277-9536(01)00291-X

47. Jacquemyn Y, Ahankour F, Martens G. Flemish obstetricians' personal preference regarding mode of delivery and attitude towards caesarean section on demand. Eur J Obstet Gynecol Reprod Biol. 2003;111(2):164-6. DOI: http://dx.doi. org/10.1016/S0301

48. Bremberg S, Nilstun T. Justifications of physicians' choice of action. Scand J Prim Health Care. 2005;23(2):102-8. DOI: http://dx.doi.org/10.1080/02813430510018455

49. Cahana A, Weibel H, Hurst SA. Ethical decision-making: do anesthesiologists, surgeons, nurse anesthetists, and surgical nurses reason similarly? Pain Med. 2008;9(6):728-36. DOI: http://dx.doi.org/10.1080/02813430510018455.

50. Ordonez Fernandez MP. Psicoterapia de Apoyo en Atención Primaria. Rev Clin Med Fam. 2008;2(5):226-35. DOI: http:// dx.doi.org/10.4321/S1699-695X2008000300006

51. Hunt O, McCurley N, Dempster M, Marley J. Patient anxiety and IV sedation in Northern Ireland. Br Dent J. 2011;210(12):5759. DOI: http://dx.doi.org/10.1038/sj.bdj.2011.483

52. Aulakh RS, Melsen B. When should orthodontics be part of reconstruction of a degenerating dentition? A case report. Prog Orthod. 2011;12(2):161-8. DOI: http://dx.doi.org/10.1016/j.pio.2011.06.006

53. Kitzinger HB, Abayev S, Pittermann A, Karle B, Kubiena H, Bohdjalian A, et al. The prevalence of body contouring surgery after gastric bypass surgery. Obes Surg. 2012;22(1):8-12. DOI: http://dx.doi.org/10.1007/s11695-011-0459-1 
54. Desplenter FA, Laekeman GJ, De Coster S; VZA Psychiatry Research Group, Simoens SR. Information on antidepressants for psychiatric inpatients: the divide between patient needs and professional practice. Pharm Pract (Granada). 2013;11(2):819. DOI: http://dx.doi.org/10.4321/S1886-36552013000200004

55. Costa FS, Bandeira DR, Trentini C, Brilmann M, Friedman R, Nunes MA. Considerações acerca da avaliação psicológica das comorbidades psiquiátricas em obesos. Psicol Est. 2009;14(2):287-93. DOl: http://dx.doi.org/10.1590/S141373722009000200009

56. Miranda Hiriart G, Saffie Gatica X. Pacientes policonsultantes: ¿un síntoma del sistema de salud en Chile? Acta Bioeth. 2014;20(2):215-23. DOI: http://dx.doi.org/10.4067/S1726-569X2014000200009

57. Nakai K, Yamamoto N, Kamei M, Fujita M. The effect of one-dose package on medication adherence for the elderly care in Japan. Pharm Pract (Granada). 2009;7(1):59-62. DOI: http://dx.doi.org/10.4321/S1886-36552009000100009

58. Burkey JM, Berenholz LP, Lippy WH; Lippy Group. Latent demand for the bone-anchored hearing aid: the Lippy Group experience. Otol Neurotol. 2006;27(5):648-52. DOI: http://dx.doi.org/10.1097/01.mao.0000224086.15968.0d

59. Packel L, Keller A, Dow WH, de Walque D, Nathan R, Mtenga S. Evolving strategies, opportunistic implementation: HIV risk reduction in Tanzania in the context of an incentive-based HIV prevention intervention. PLoS One. 2012;7(8):e44058. DOI: http://dx.doi.org/10.1371/journal.pone.0044058

60. Gross R, McNeill R, Davis P, Lay-Yee R, Jatrana S, Crampton P. The association of gender concordance and primary care physicians' perceptions of their patients. Women Health. 2008;48(2):123-44. DOI: http://dx. doi. org/10.1080/03630240802313464

61. Fallon D, Warne T, McAndrew S, McLaughlin H. An adult education: learning and understanding what young service users and carers really, really want in terms of their mental well being. Nurse Educ Today. 2012;32(2):128-32. DOI: http://dx.doi. org/10.1016/j.nedt.2011.06.002

62. Tempark T, Shwayder T. Chinese fortune-telling based on face and body mole positions: a hidden agenda regarding mole removal. Arch Dermatol. 2012;148(6):772-3. DOI: http://dx.doi.org/10.1001/archdermatol.2012.949

63. McLeod ME. The caring physician: a journey in self-exploration and self-care. Am J Gastroenterol. 2003;98(10):2135-8. PMID: 14572557 DOI: http://dx.doi.org/10.1111/j.1572-0241.2003.07719.x

64. Towersey L, Sinclair J. Collins Cobuild student's dictionary: bridge bilingual Portuguese. London: Harper Collins Publishers; 1995.

65. Courtenay WH. Constructions of masculinity and their influence on men's well-being: a theory of gender and health. Soc Sci Med. 2000;50(10):1385-401. DOI: http://dx.doi.org/10.1016/S0277-9536(99)00390-1

66. Norman $A H$, Tesser $C D$. Prevenção quaternária na atenção primária à saúde: uma necessidade do Sistema Único de Saúde. Cad Saúde Pública. 2009;25(9):2012-20. DOI: http://dx.doi.org/10.1590/S0102-311X2009000900015

67. Gusso G, Poli Neto P. Gestão da Clínica. In: Gusso G, Lopes JMC, orgs. Tratado de medicina de família e comunidade: princípios, formação e prática. Porto Alegre: Artmed; 2012. p.159-66.

68. Schraiber LB, Mendes-Gonçalves RB. Necessidades de saúde e atenção primária. In: Schraiber LB, Nemes MIB, MendesGonçalves RB, orgs. Saúde do adulto: programas e ações na unidade básica. 2a ed. São Paulo: HUCITEC; 2000. p.29-47.

69. Rocha F. Algumas considerações sobre as entrevistas preliminares, demanda e início de análise. Percurso. $1991 ; 3(5 / 6): 15-21$. 